\title{
WATER STORAGE AND THE MOVEMENTS OF BODY FLUIDS AND CHLORIDES DURING ACUTE LIVER DISEASE
}

\author{
By DANIEL H. LABBY, AND CHARLES L. HOAGLAND 1 \\ (From the Hospital of The Rockefeller Institute for Medical Research)
}

(Received for publication December 9, 1946)

The role of the liver in directing fluid shifts in the body has never been accurately defined. The effect of hepatic disease on the water balance of the body, however, has been appreciated for many years. In evidence of this is the fact that a patient with liver disease experiences greater delay in diuresis than does a normal person after drinking large amounts of water (1). The suppression of urine that may accompany acute liver disease as well as the oliguria of cirrhosis are old clinical observations $(1,2,3,4,5)$. The phenomenon of water storage during acute hepatic disease was suggested by Jones and Eaton (6) who pointed out that the convalescent phase of acute hepatitis was frequently initiated by diuresis which they assumed was a result of the mobilization of previously stored water. Since reasonably reliable methods exist for the measurement of individual body fluid spaces and have been applied with success to the study of other infections (7), it was thought that a systematic study of the distribution of body water during the course of acute infectious hepatitis would reveal the presence of water storage and would further serve to identify the individual fluid compartments participating. In addition; periodic measurements of separate fluid shifts during the course of the disease would give quantitative expression to the relative role played by each fluid compartment while operating under hepatic control.

In a previous communication (8) data from one typical case were presented. However, in observing the fluid alterations in a group of 14 patients with acute infectious hepatitis, the movements of body water in each case were found to follow a pattern of great similarity. It was, therefore, thought that a presentation of consolidated data from the entire study would serve to emphasize the uniformity of these phenomena.

The present study was concerned with 14 young adult males who were part of a large group of

1 Dr. Hoagland died August 2, 1946.
Navy personnel with acute infectious hepatitis observed at the Hospital of The Rockefeller Institute for Medical Research (9). Each patient had been previously healthy and had developed hepatitis during active naval service. Only those cases in the early acute phase of hepatitis were included in the present study. Since water and electrolyte measurements were of primary interest, it was thought advisable to study only those patients who had been able to maintain a fluid intake approximating normal and in whom diarrhea, vomiting, severe fever, and dehydration were not complicating features of the acute phase of illness. The course of disease was comparable in each patient, being of moderate severity and proceeding in each instance through uncomplicated convalescence to complete clinical healing (10).

\section{METHODS}

On admission to the hospital, each patient was placed at bed rest in the Metabolic Ward where the entire study was conducted. The diagnosis of acute infectious hepatitis was readily established after a careful medical history and physical examination, and tests of liver function served to confirm the presence of acute liver disease in each instance. A urine examination done on admission and repeated frequently during the hospital course ruled out underlying renal disease in each case. Strict control of water balance was established by measurements of fluid intake and urine output and daily determinations of body weight. No patient received additional fluid by clysis. The entire group received a diet composed of 150 grams of protein, 50 grams of fat, and sufficient carbohydrate to maintain the daily food intake between 3,000 and 3,500 calories. The total daily salt intake including the calculated intrinsic food salt was kept.constantly between 9 and 10 grams.

The initial measurements of plasma volume, blood volume, and "extravascular thiocyanate space" were begun under basal conditions on the day following admission to the hospital and repeated at least every 7 days, occasionally as often as every 4 days. The simultaneous method of Gregersen and Stewart (11), based upon the plasma dilution of the dye T-1824 and the "extravascular space" dilution of sodium thiocyanate, was adapted to the Coleman Jr. spectrophotometer. The selectivity of this instrument minimized the theoretical effect of the inter- 
ference of bilirubin and hemolysis (12) on the determination of T-1824. Dye-free samples of serum obtained before injection of $\mathrm{T}-1824$ were used for control readings. Many preliminary tests on other patients with hepatitis indicated that 15 minutes was an appropriate interval for the "mixing time" of the dye in each case (13). All blood samples were obtained without the use' of a tourniquet.

In the present study the "extravascular thiocyanate space" was derived by subtracting the plasma volume from the total thiocyanate space. So little change was encountered in the total red blood cell volume as expressed by the venous hematocrit (14) that small variations in the amount of red cell water were not considered of importance for these calculations (11). The total blood volume was calculated from simultaneous determinations of the plasma volume and venous hematocrit according to the formula (15):

$$
\frac{\text { Plasma volume }}{(100-\text { hematocrit })} \times 100=\text { Blood volume. }
$$

The measurements of all body fluid compartments were finally expressed on a per kilogram of body weight basis according to previously described standards (16). No measurements of the intracellular fluid phase were undertaken.

It had been previously determined (9) that the most reliable index of liver function during the course of acute infectious hepatitis is a combination of measurements of the total serum proteins with estimations of the albumin and globulin fractions, the plasma bilirubin level, the bromsulfalein excretion test $(5 \mathrm{mgm}$. per $\mathrm{kgm}$.-45-

TABLE I

Alterations in body fluids, the components of blood and urine, and tests of liver function during acute infectious hepatitis

\begin{tabular}{|c|c|c|c|c|c|c|c|c|c|c|c|c|c|c|c|c|c|c|c|c|}
\hline \multirow{2}{*}{$\begin{array}{c}\text { Case } \\
\text { no. }\end{array}$} & \multirow{2}{*}{ Age } & \multirow{2}{*}{$\begin{array}{l}\text { Day } \\
\text { of } \\
\text { dis- } \\
\text { ease }\end{array}$} & \multirow{2}{*}{$\begin{array}{c}\text { Day } \\
\text { since } \\
\text { onset } \\
\text { dark } \\
\text { urine }\end{array}$} & \multirow{2}{*}{$\begin{array}{c}\text { Body } \\
\text { wt. }\end{array}$} & \multirow{2}{*}{$\begin{array}{c}\text { He- } \\
\text { mato- } \\
\text { crit }\end{array}$} & \multirow{2}{*}{$\begin{array}{c}\text { Thio- } \\
\text { cya- } \\
\text { nate } \\
\text { space }\end{array}$} & \multirow{2}{*}{$\begin{array}{c}\text { Plasma } \\
\text { vol. }\end{array}$} & \multirow{2}{*}{$\begin{array}{l}\text { Blood } \\
\text { vol. }\end{array}$} & \multicolumn{4}{|c|}{ Plasma proteins } & \multirow{2}{*}{ NPN } & \multirow{2}{*}{$\begin{array}{l}\text { Se- } \\
\text { rum } \\
\text { bili- } \\
\text { ru- } \\
\text { bin }\end{array}$} & \multirow{2}{*}{$\begin{array}{l}\text { Brom- } \\
\text { sulfa- } \\
\text { lein } \\
\text { reten- } \\
\text { tion* }\end{array}$} & \multirow{2}{*}{$\begin{array}{l}\text { Thy- } \\
\text { mol } \\
\text { tur- } \\
\text { bid- } \\
\text { ity }\end{array}$} & \multirow{2}{*}{$\begin{array}{l}\text { Water } \\
\text { toler- } \\
\text { ance } \\
\text { test } f\end{array}$} & \multicolumn{3}{|c|}{ Chlorides } \\
\hline & & & & & & & & & Total & $\begin{array}{l}\text { Al- } \\
\text { bu- } \\
\text { min }\end{array}$ & $\begin{array}{l}\text { Glob- } \\
\text { ulin }\end{array}$ & $A / G$ & & & & & & Plasma & Uri & \\
\hline 1 & 32 & $\begin{array}{r}7 \\
13 \\
20 \\
27 \\
34\end{array}$ & $\begin{array}{r}2 \\
8 \\
15 \\
22 \\
29\end{array}$ & \begin{tabular}{l|} 
kgm. \\
\\
80.4 \\
79.4 \\
78.6 \\
77.8 \\
78.0
\end{tabular} & $\begin{array}{l}51 \\
51 \\
47 \\
50\end{array}$ & $\begin{array}{c}\text { ml. per } \\
\text { kgm. } \\
\text { body } \\
w t . \\
200 \\
186 \\
175 \\
170\end{array}$ & $\begin{array}{c}\text { ml. per } \\
\text { kgm. } \\
\text { body } \\
w t . \\
42 \\
40 \\
37 \\
32\end{array}$ & $\begin{array}{c}\text { ml. per } \\
\text { kgm. } \\
\text { body } \\
\text { wt. } \\
85 \\
81 \\
70 \\
62\end{array}$ & $\begin{array}{l}6.42 \\
7.31 \\
7.11 \\
7.32 \\
7.36\end{array}$ & $\begin{array}{l}3.30 \\
3.87 \\
3.90 \\
3.99 \\
4.28\end{array}$ & $\begin{array}{l}3.12 \\
3.44 \\
3.21 \\
3.33 \\
3.08\end{array}$ & $\begin{array}{l}1.1 \\
1.1 \\
1.2 \\
1.2 \\
1.4\end{array}$ & $\begin{array}{c}m g m . \\
\text { per } \\
\text { cent } \\
21.2 \\
30.0 \\
36.2 \\
40.0 \\
35.0\end{array}$ & $\begin{array}{c}\text { mgm. } \\
\text { per } \\
\text { cent } \\
6.6 \\
4.2 \\
1.9 \\
1.3 \\
1.0\end{array}$ & $\begin{array}{l}\text { per } \\
\text { cent } \\
\\
43.0 \\
31.0 \\
16.5 \\
10.0\end{array}$ & \begin{tabular}{c|} 
units \\
\\
23 \\
24 \\
19 \\
15 \\
11
\end{tabular} & $\begin{array}{r}794 \\
1,272 \\
1,296 \\
1,307 \\
1,446\end{array}$ & $\begin{array}{c}\text { m. eq. } \\
\text { conc. } \\
\text { per } \\
\text { liter } \\
95.8 \\
97.8 \\
99.8 \\
99.8 \\
101.0\end{array}$ & $\begin{array}{c}\text { m. eq. } \\
\text { conc. } \\
\text { per } \\
\text { liter } \\
27.8 \\
66.8 \\
55.8 \\
52.8 \\
97.8\end{array}$ & $\begin{array}{l}\text { m. eq. } \\
24 \text { hr. } \\
\text { out- } \\
\text { put } \\
114 \\
193 \\
167 \\
153 \\
169\end{array}$ \\
\hline 2 & 17 & $\begin{array}{r}6 \\
8 \\
15 \\
22 \\
\end{array}$ & $\begin{array}{r}3 \\
5 \\
12 \\
19\end{array}$ & $\begin{array}{l}\mathbf{5 6 . 2} \\
\mathbf{5 6 . 6} \\
\mathbf{5 8 . 1} \\
\mathbf{5 9 . 3}\end{array}$ & $\begin{array}{l}48 \\
47 \\
45 \\
44\end{array}$ & $\begin{array}{l}218 \\
204 \\
201 \\
197\end{array}$ & $\begin{array}{l}56 \\
41 \\
44 \\
38\end{array}$ & $\begin{array}{r}107 \\
78 \\
80 \\
67\end{array}$ & $\begin{array}{l}6.54 \\
6.47 \\
7.86\end{array}$ & $\begin{array}{l}4.26 \\
3.96 \\
4.76\end{array}$ & $\begin{array}{l}2.28 \\
2.51 \\
3.10\end{array}$ & $\begin{array}{l}1.9 \\
1.6 \\
1.6\end{array}$ & $\begin{array}{l}28.7 \\
26.2 \\
28.7\end{array}$ & $\begin{array}{l}4.0 \\
2.2 \\
0.7 \\
0.5\end{array}$ & $\begin{array}{l}34.5 \\
10.5 \\
11.5\end{array}$ & $\begin{array}{r}13 \\
13 \\
12 \\
6\end{array}$ & $\begin{array}{l}1,183 \\
1,305 \\
1,572\end{array}$ & $\begin{array}{r}96.8 \\
100.0\end{array}$ & $\begin{array}{l}56.8 \\
71.6\end{array}$ & $\begin{array}{l}165 \\
145\end{array}$ \\
\hline 3 & 19 & $\begin{array}{r}4 \\
11 \\
18 \\
25 \\
32 \\
49\end{array}$ & $\begin{array}{r}3 \\
10 \\
17 \\
24 \\
31 \\
48\end{array}$ & $\begin{array}{l}78.3 \\
77.7 \\
76.8 \\
77.0 \\
77.8 \\
77.7\end{array}$ & $\begin{array}{l}51 \\
51 \\
52 \\
50 \\
49 \\
49\end{array}$ & $\begin{array}{l}169 \\
148 \\
148 \\
149 \\
151 \\
155\end{array}$ & $\begin{array}{l}45 \\
44 \\
50 \\
50 \\
49 \\
47\end{array}$ & $\begin{array}{r}91 \\
90 \\
104 \\
99 \\
96 \\
92\end{array}$ & $\begin{array}{l}6.10 \\
7.10 \\
7.20 \\
6.65 \\
8.01 \\
6.49\end{array}$ & $\begin{array}{l}3.40 \\
3.64 \\
4.45 \\
4.22 \\
4.94 \\
4.10\end{array}$ & $\begin{array}{l}2.70 \\
3.46 \\
2.75 \\
2.43 \\
3.07 \\
2.39\end{array}$ & $\begin{array}{l}1.3 \\
1.1 \\
1.6 \\
1.7 \\
1.6 \\
1.7\end{array}$ & $\begin{array}{l}36.2 \\
37.5 \\
35.0 \\
35.0 \\
30.0 \\
23.7\end{array}$ & $\begin{array}{l}6.9 \\
1.6 \\
1.3 \\
0.4 \\
0.8 \\
0.4\end{array}$ & $\begin{array}{r}45.0 \\
4.5 \\
7.5 \\
2.5 \\
1.0 \\
1.2\end{array}$ & $\begin{array}{r}17 \\
30 \\
20 \\
21 \\
14 \\
8\end{array}$ & \begin{tabular}{r|}
519 \\
1,037 \\
1,459 \\
1,726 \\
1,817 \\
\end{tabular} & \begin{tabular}{r|}
93.0 \\
95.8 \\
99.8 \\
102.0 \\
102.0 \\
101.0
\end{tabular} & $\begin{array}{r}18.8 \\
61.8 \\
93.0 \\
82.0 \\
128.0 \\
142.0\end{array}$ & $\begin{array}{r}42 \\
173 \\
273 \\
225 \\
183 \\
216\end{array}$ \\
\hline 4 & 25 & $\begin{array}{r}8 \\
15 \\
23 \\
30 \\
47\end{array}$ & $\begin{array}{r}4 \\
11 \\
19 \\
26 \\
43\end{array}$ & $\begin{array}{l}75.7 \\
75.3 \\
76.3 \\
77.6 \\
79.1\end{array}$ & $\begin{array}{l}51 \\
51 \\
49 \\
47 \\
50\end{array}$ & $\begin{array}{l}190 \\
185 \\
170 \\
162 \\
170\end{array}$ & $\begin{array}{l}52 \\
40 \\
45 \\
45 \\
41\end{array}$ & $\begin{array}{r}107 \\
82 \\
88 \\
85 \\
82\end{array}$ & $\begin{array}{l}5.61 \\
6.35 \\
6.66 \\
6.94 \\
6.55\end{array}$ & $\begin{array}{l}3.56 \\
3.67 \\
4.61 \\
4.77 \\
4.03\end{array}$ & $\begin{array}{l}2.05 \\
2.68 \\
2.05 \\
2.17 \\
2.52\end{array}$ & $\begin{array}{l}1.7 \\
1.4 \\
2.2 \\
2.2 \\
1.6\end{array}$ & $\begin{array}{l}25.0 \\
32.5 \\
26.2 \\
26.2\end{array}$ & $\begin{array}{l}4.9 \\
1.6 \\
0.8 \\
0.6 \\
0.6\end{array}$ & $\begin{array}{r}39.5 \\
3.8 \\
0.5 \\
0.5 \\
0.5\end{array}$ & $\begin{array}{r}20 \\
19 \\
12 \\
9 \\
8\end{array}$ & $\begin{array}{r}439 \\
947 \\
1,218 \\
1,618\end{array}$ & $\begin{array}{r}93.8 \\
98.8 \\
99.8 \\
101.0\end{array}$ & $\begin{array}{l}36.8 \\
52.0 \\
76.0 \\
80.0\end{array}$ & $\begin{array}{r}79 \\
126 \\
130 \\
173\end{array}$ \\
\hline 5 & 20 & $\begin{array}{r}7 \\
15 \\
22 \\
29 \\
36 \\
51\end{array}$ & $\begin{array}{r}4 \\
12 \\
19 \\
26 \\
33 \\
48\end{array}$ & $\begin{array}{l}63.5 \\
64.7 \\
65.0 \\
66.0 \\
67.4 \\
66.9\end{array}$ & $\begin{array}{l}50 \\
49 \\
48 \\
48 \\
47\end{array}$ & $\begin{array}{l}258 \\
234 \\
216 \\
210 \\
202\end{array}$ & $\begin{array}{l}43 \\
43 \\
41 \\
38 \\
36\end{array}$ & $\begin{array}{l}86 \\
83 \\
79 \\
73 \\
68\end{array}$ & $\begin{array}{l}5.91 \\
6.81 \\
7.59 \\
7.25 \\
7.46 \\
6.56\end{array}$ & $\begin{array}{l}3.08 \\
4.36 \\
4.34 \\
4.69 \\
5.06 \\
4.40\end{array}$ & $\begin{array}{l}2.83 \\
2.45 \\
3.25 \\
2.56 \\
2.40 \\
2.16\end{array}$ & $\begin{array}{l}1.1 \\
1.8 \\
1.3 \\
1.8 \\
2.1 \\
2.0\end{array}$ & $\begin{array}{l}26.3 \\
22.5 \\
35.0 \\
38.7 \\
30.0 \\
25.0\end{array}$ & $\begin{array}{l}9.7 \\
2.8 \\
1.6 \\
1.0 \\
0.8 \\
0.5\end{array}$ & $\begin{array}{r}39.5 \\
21.5 \\
21.5 \\
10.0 \\
4.0 \\
0.0\end{array}$ & $\begin{array}{l}25 \\
29 \\
20 \\
16 \\
16 \\
12\end{array}$ & $\begin{array}{l}1,251 \\
1,248 \\
1,205 \\
1,344 \\
1,221 \\
1,222\end{array}$ & $\begin{array}{r}99.0 \\
103.0 \\
100.8 \\
101.0 \\
101.0\end{array}$ & $\begin{array}{r}53.0 \\
96.0 \\
82.0 \\
73.0 \\
101.0\end{array}$ & $\begin{array}{l}171 \\
241 \\
162 \\
148 \\
172\end{array}$ \\
\hline 6 & 17 & $\begin{array}{r}7 \\
15 \\
23 \\
30 \\
37 \\
53 \\
\end{array}$ & $\begin{array}{r}6 \\
14 \\
22 \\
29 \\
36 \\
52 \\
\end{array}$ & $\begin{array}{l}66.2 \\
66.3 \\
67.6 \\
69.4 \\
70.5 \\
72.1\end{array}$ & $\begin{array}{l}49 \\
49 \\
50 \\
48 \\
47 \\
47\end{array}$ & $\begin{array}{l}182 \\
170 \\
176 \\
174 \\
183 \\
180 \\
\end{array}$ & $\begin{array}{l}58 \\
44 \\
40 \\
38 \\
39 \\
40\end{array}$ & $\begin{array}{r}113 \\
86 \\
81 \\
73 \\
73 \\
74\end{array}$ & $\begin{array}{l}6.73 \\
6.81 \\
7.24 \\
7.17 \\
7.03 \\
6.45\end{array}$ & $\begin{array}{l}4.34 \\
4.47 \\
4.70 \\
4.71 \\
4.84 \\
4.25\end{array}$ & $\begin{array}{l}2.39 \\
2.34 \\
2.54 \\
2.46 \\
2.19 \\
2.20\end{array}$ & $\begin{array}{l}1.8 \\
1.5 \\
1.6 \\
1.9 \\
2.2 \\
1.9\end{array}$ & $\begin{array}{l}35.0 \\
35.0 \\
27.5 \\
26.2 \\
25.0 \\
30.0\end{array}$ & $\begin{array}{l}2.9 \\
1.4 \\
1.2 \\
0.8 \\
0.7 \\
0.5\end{array}$ & $\begin{array}{r}29.5 \\
23.0 \\
8.5 \\
3.7 \\
2.5 \\
2.5\end{array}$ & $\begin{array}{r}11 \\
9 \\
7 \\
4 \\
4 \\
2\end{array}$ & $\begin{array}{l}1,167 \\
1,329 \\
1,210 \\
1,153 \\
1,117 \\
1,302 \\
\end{array}$ & $\begin{array}{r}95.0 \\
98.8 \\
101.0 \\
101.0 \\
101.0\end{array}$ & $\begin{array}{r}54.0 \\
109.0 \\
113.0 \\
96.8 \\
116.0\end{array}$ & $\begin{array}{l}136 \\
167 \\
213 \\
177 \\
188\end{array}$ \\
\hline 7 & 19 & $\begin{array}{l}10 \\
18 \\
25 \\
31 \\
38 \\
52\end{array}$ & $\begin{array}{r}6 \\
14 \\
21 \\
27 \\
34 \\
48\end{array}$ & $\begin{array}{l}59.5 \\
60.1 \\
60.8 \\
62.1 \\
63.4 \\
63.0\end{array}$ & $\begin{array}{l}46 \\
46 \\
46 \\
46 \\
46 \\
45\end{array}$ & $\begin{array}{l}198 \\
189 \\
181 \\
183 \\
185 \\
186\end{array}$ & $\begin{array}{l}49 \\
52 \\
57 \\
45 \\
41 \\
47\end{array}$ & $\begin{array}{r}92 \\
95 \\
106 \\
83 \\
75 \\
85\end{array}$ & $\begin{array}{l}6.58 \\
6.73 \\
6.40 \\
6.76 \\
6.78 \\
7.29\end{array}$ & $\begin{array}{l}3.91 \\
3.89 \\
4.21 \\
4.64 \\
4.83 \\
4.54\end{array}$ & $\begin{array}{l}2.67 \\
2.84 \\
2.19 \\
2.12 \\
1.95 \\
2.75\end{array}$ & $\begin{array}{l}1.5 \\
1.4 \\
1.9 \\
2.0 \\
2.5 \\
1.7\end{array}$ & $\begin{array}{l}33.7 \\
18.7 \\
26.2 \\
32.5\end{array}$ & $\begin{array}{l}7.1 \\
1.6 \\
1.0 \\
0.7 \\
0.9 \\
0.8\end{array}$ & $\begin{array}{r}\mathbf{5 0 . 5} \\
\mathbf{3 . 5} \\
\mathbf{4 . 5} \\
\mathbf{2 . 0} \\
0.5 \\
1.2\end{array}$ & $\begin{array}{r}14 \\
7 \\
5 \\
8 \\
4 \\
4\end{array}$ & $\begin{array}{r}392 \\
1,584 \\
1,709 \\
1,524 \\
1,698 \\
1,536\end{array}$ & \begin{tabular}{r|}
98.2 \\
99.8 \\
102.0 \\
100.0 \\
103.0
\end{tabular} & $\begin{array}{r}48.2 \\
74.0 \\
113.0 \\
95.0 \\
111.0 \\
137.0\end{array}$ & $\begin{array}{r}66 \\
186 \\
241 \\
214 \\
276 \\
218\end{array}$ \\
\hline 8 & 21 & $\begin{array}{r}7 \\
10 \\
18 \\
25 \\
33 \\
45\end{array}$ & $\begin{array}{r}6 \\
9 \\
17 \\
24 \\
32 \\
44\end{array}$ & $\begin{array}{l}49.6 \\
49.5 \\
49.6 \\
51.5 \\
52.6 \\
53.4\end{array}$ & $\begin{array}{l}48 \\
48 \\
47 \\
48 \\
46 \\
46\end{array}$ & $\begin{array}{l}245 \\
239 \\
216 \\
215 \\
205 \\
217\end{array}$ & $\begin{array}{l}43 \\
42 \\
43 \\
35 \\
36 \\
36\end{array}$ & $\begin{array}{l}83 \\
80 \\
80 \\
67 \\
67 \\
66\end{array}$ & $\begin{array}{l}7.60 \\
6.82 \\
7.36 \\
7.78 \\
7.64 \\
7.32\end{array}$ & $\begin{array}{l}4.04 \\
4.23 \\
3.98 \\
4.56 \\
4.71 \\
4.70\end{array}$ & $\begin{array}{l}3.56 \\
2.59 \\
3.38 \\
3.22 \\
2.93 \\
2.62\end{array}$ & $\begin{array}{l}1.1 \\
1.6 \\
1.2 \\
1.4 \\
1.6 \\
1.8\end{array}$ & $\begin{array}{l}32.5 \\
32.5 \\
35.0 \\
30.0 \\
26.3 \\
27.5\end{array}$ & $\begin{array}{l}7.3 \\
8.3 \\
1.8 \\
0.8 \\
1.0 \\
0.7\end{array}$ & $\begin{array}{r}41.0 \\
7.5 \\
4.5 \\
7.5 \\
1.0\end{array}$ & $\begin{array}{r}13 \\
20 \\
14 \\
12 \\
7 \\
4\end{array}$ & $\begin{array}{r}775 \\
1,346 \\
1,341 \\
1,343 \\
1,326\end{array}$ & $\begin{array}{r}95.0 \\
94.0 \\
102.0 \\
103.0 \\
101.0 \\
102.0\end{array}$ & $\begin{array}{r}46.0 \\
80.0 \\
110.0 \\
89.0\end{array}$ & $\begin{array}{l}147 \\
163 \\
223 \\
165\end{array}$ \\
\hline
\end{tabular}


TABLE I-Continued

\begin{tabular}{|c|c|c|c|c|c|c|c|c|c|c|c|c|c|c|c|c|c|c|c|c|}
\hline \multirow{2}{*}{$\begin{array}{c}\text { Case } \\
\text { no. }\end{array}$} & \multirow{2}{*}{ Age } & \multirow{2}{*}{$\begin{array}{l}\text { Day } \\
\text { of } \\
\text { dis- } \\
\text { ease }\end{array}$} & \multirow{2}{*}{$\begin{array}{c}\text { Day } \\
\text { since } \\
\text { onset } \\
\text { dark } \\
\text { urine }\end{array}$} & \multirow{2}{*}{$\begin{array}{c}\text { Body } \\
\text { wt. }\end{array}$} & \multirow{2}{*}{$\begin{array}{c}\text { He- } \\
\text { mato- } \\
\text { crit }\end{array}$} & \multirow{2}{*}{$\begin{array}{l}\text { Thio- } \\
\text { cya- } \\
\text { nate } \\
\text { space }\end{array}$} & \multirow{2}{*}{$\begin{array}{c}\text { Plasma } \\
\text { vol. }\end{array}$} & \multirow{2}{*}{$\begin{array}{l}\text { Blood } \\
\text { vol. }\end{array}$} & \multicolumn{4}{|c|}{ Plasma proteins } & \multirow{2}{*}{ NPN } & \multirow{2}{*}{$\begin{array}{l}\text { Se- } \\
\text { rum } \\
\text { bili- } \\
\text { ru- } \\
\text { bin }\end{array}$} & \multirow{2}{*}{$\begin{array}{l}\text { Brom- } \\
\text { sulfa- } \\
\text { lein } \\
\text { reten- } \\
\text { tion* }\end{array}$} & \multirow{2}{*}{$\begin{array}{l}\text { Thy- } \\
\text { mol } \\
\text { tur- } \\
\text { bid- } \\
\text { ity }\end{array}$} & \multirow{2}{*}{$\begin{array}{l}\text { Water } \\
\text { toler- } \\
\text { ance } \\
\text { test } \dagger\end{array}$} & \multicolumn{3}{|c|}{ Chlorides } \\
\hline & & & & & & & & & Total & $\begin{array}{l}\text { Al- } \\
\text { bu- }\end{array}$ & Glob- & A/G & & & & & & Plasma & Uri & \\
\hline 9 & 25 & $\begin{array}{l}15 \\
24 \\
31 \\
38 \\
45 \\
50\end{array}$ & $\begin{array}{r}6 \\
15 \\
22 \\
29 \\
36 \\
41\end{array}$ & \begin{tabular}{|l|}
$\mathrm{kgm}$. \\
\\
81.4 \\
80.0 \\
79.6 \\
79.4 \\
79.6 \\
79.8
\end{tabular} & $\begin{array}{l}46 \\
44 \\
45 \\
48 \\
47\end{array}$ & $\begin{array}{c}\text { ml. per } \\
\text { kgm. } \\
\text { body } \\
\text { wt. } \\
218 \\
191 \\
193 \\
195 \\
192\end{array}$ & $\begin{array}{c}\text { ml. per } \\
\text { kgm. } \\
\text { body } \\
w t . \\
45 \\
41 \\
40 \\
40 \\
40\end{array}$ & $\begin{array}{c}\text { ml. per } \\
\text { kgm. } \\
\text { body } \\
\text { wt. } \\
83 \\
73 \\
72 \\
76 \\
76\end{array}$ & $\begin{array}{l}6.81 \\
7.32 \\
6.98 \\
7.61 \\
6.91 \\
6.23\end{array}$ & $\begin{array}{l}3.99 \\
4.07 \\
4.17 \\
4.36 \\
4.57 \\
4.26\end{array}$ & $\begin{array}{l}2.82 \\
3.25 \\
2.81 \\
3.25 \\
2.34 \\
1.97\end{array}$ & $\begin{array}{l}1.4 \\
1.3 \\
1.5 \\
1.3 \\
1.9 \\
2.2\end{array}$ & $\begin{array}{c}\text { mgm. } \\
\text { per } \\
\text { cent } \\
21.2 \\
28.7 \\
32.5 \\
31.2 \\
28.7 \\
27.5\end{array}$ & $\begin{array}{c}\text { mgm. } \\
\text { per } \\
\text { cent } \\
5.3 \\
1.4 \\
1.0 \\
0.9 \\
0.7 \\
0.5\end{array}$ & $\begin{array}{r}\begin{array}{c}\text { per } \\
\text { cent }\end{array} \\
\\
38.5 \\
5.0 \\
3.5 \\
2.0 \\
2.0 \\
2.0\end{array}$ & $\begin{array}{r}\text { units } \\
\\
23 \\
12 \\
13 \\
12 \\
8 \\
9\end{array}$ & $\begin{array}{l}1,282 \\
1,451 \\
1,483 \\
1,545 \\
1,548 \\
1,498\end{array}$ & $\begin{array}{c}\text { m. eq. } \\
\text { conc. } \\
\text { per } \\
\text { liter } \\
96.8 \\
101.0 \\
103.0 \\
104.0 \\
107.0\end{array}$ & $\begin{array}{c}\text { m. eq. } \\
\text { conc. } \\
\text { per } \\
\text { liter } \\
50.0 \\
92.0 \\
82.0 \\
87.8\end{array}$ & $\begin{array}{c}\text { m. eq. } \\
24 \text { hr. } \\
\text { out- } \\
\text { put } \\
129 \\
135 \\
125 \\
98\end{array}$ \\
\hline 10 & 34 & $\begin{array}{l}12 \\
16 \\
23 \\
30 \\
36 \\
51\end{array}$ & $\begin{array}{r}7 \\
11 \\
18 \\
25 \\
31 \\
46\end{array}$ & $\begin{array}{l}\mathbf{5 6 . 6} \\
\mathbf{5 6 . 0} \\
\mathbf{5 6 . 6} \\
\mathbf{5 7 . 1} \\
\mathbf{5 7 . 0} \\
\mathbf{5 7 . 4}\end{array}$ & $\begin{array}{l}46 \\
46 \\
45 \\
45 \\
47 \\
47\end{array}$ & $\begin{array}{l}242 \\
224 \\
223 \\
216 \\
217 \\
219\end{array}$ & $\begin{array}{l}49 \\
46 \\
45 \\
40 \\
40 \\
41\end{array}$ & $\begin{array}{l}90 \\
85 \\
82 \\
72 \\
75 \\
77\end{array}$ & $\begin{array}{l}6.25 \\
5.85 \\
6.23 \\
6.24 \\
7.33 \\
6.85\end{array}$ & $\begin{array}{l}3.97 \\
4.09 \\
4.07 \\
4.21 \\
4.83 \\
4.70\end{array}$ & $\begin{array}{l}2.28 \\
1.76 \\
2.16 \\
2.03 \\
2.50 \\
2.15\end{array}$ & $\begin{array}{l}1.7 \\
2.3 \\
1.9 \\
2.1 \\
1.5 \\
2.2\end{array}$ & $\begin{array}{l}25.0 \\
25.0 \\
28.7 \\
26.2 \\
26.3 \\
28.7\end{array}$ & $\begin{array}{l}5.0 \\
5.0 \\
1.9 \\
0.9 \\
1.0 \\
0.6\end{array}$ & $\begin{array}{r}35.5 \\
33.5 \\
8.0 \\
10.0 \\
5.0 \\
6.0\end{array}$ & $\begin{array}{l}21 \\
21 \\
17 \\
13 \\
14 \\
10\end{array}$ & $\begin{array}{r}503 \\
1,170 \\
1,358 \\
1,341 \\
1,529\end{array}$ & $\begin{array}{r}97.0 \\
102.0 \\
101.0 \\
103.0 \\
102.0 \\
103.0\end{array}$ & $\begin{array}{l}17.0 \\
41.0 \\
27.0 \\
47.0 \\
45.0\end{array}$ & $\begin{array}{r}55 \\
92 \\
116 \\
144 \\
134\end{array}$ \\
\hline 11 & 22 & $\begin{array}{l}10 \\
20 \\
26 \\
33 \\
37\end{array}$ & $\begin{array}{l}10 \\
20 \\
26 \\
33 \\
37\end{array}$ & $\begin{array}{l}85.8 \\
83.8 \\
84.0 \\
82.8 \\
82.4\end{array}$ & $\begin{array}{l}51 \\
50 \\
49 \\
47 \\
48\end{array}$ & $\begin{array}{l}200 \\
187 \\
182 \\
166 \\
163\end{array}$ & $\begin{array}{l}41 \\
43 \\
38 \\
39 \\
36\end{array}$ & $\begin{array}{l}83 \\
85 \\
74 \\
73 \\
69\end{array}$ & $\begin{array}{l}5.79 \\
6.91 \\
7.46 \\
7.06 \\
6.51\end{array}$ & $\begin{array}{l}3.55 \\
3.94 \\
4.25 \\
4.43 \\
4.54\end{array}$ & $\begin{array}{l}2.24 \\
2.97 \\
3.21 \\
2.63 \\
1.97\end{array}$ & $\begin{array}{l}1.6 \\
1.3 \\
1.3 \\
1.7 \\
2.3\end{array}$ & \begin{tabular}{l|}
21.2 \\
18.7 \\
30.0 \\
31.2 \\
32.5
\end{tabular} & $\begin{array}{l}7.8 \\
3.4 \\
1.9 \\
1.3 \\
1.2\end{array}$ & \begin{tabular}{r|}
43.0 \\
11.0 \\
10.0 \\
4.5 \\
2.5
\end{tabular} & $\begin{array}{l}16 \\
21 \\
15 \\
10 \\
10\end{array}$ & $\begin{array}{r}601 \\
1,520 \\
1,367 \\
1,407 \\
1,391\end{array}$ & $\begin{array}{r}90.8 \\
96.0 \\
99.0 \\
99.0 \\
103.0\end{array}$ & $\begin{array}{r}27.8 \\
107.0 \\
139.0 \\
103.0 \\
139.0\end{array}$ & $\begin{array}{r}55 \\
171 \\
200 \\
132 \\
204\end{array}$ \\
\hline 12 & 23 & $\begin{array}{l}17 \\
27 \\
33 \\
40 \\
47 \\
59\end{array}$ & $\begin{array}{l}10 \\
20 \\
26 \\
33 \\
40 \\
52\end{array}$ & $\begin{array}{l}\mathbf{5 7 . 3} \\
57.7 \\
57.0 \\
58.5 \\
59.7 \\
61.3\end{array}$ & $\begin{array}{l}47 \\
47 \\
46 \\
46 \\
47 \\
51\end{array}$ & $\begin{array}{l}194 \\
193 \\
198 \\
192\end{array}$ & $\begin{array}{l}45 \\
47 \\
54 \\
49 \\
40 \\
40\end{array}$ & $\begin{array}{r}84 \\
89 \\
100 \\
91 \\
75 \\
81\end{array}$ & $\begin{array}{l}7.23 \\
6.90 \\
6.97 \\
6.93 \\
6.89 \\
7.31\end{array}$ & $\begin{array}{l}3.75 \\
4.45 \\
4.40 \\
4.59 \\
4.86 \\
5.31\end{array}$ & $\begin{array}{l}3.48 \\
2.45 \\
2.57 \\
2.34 \\
2.03 \\
2.00\end{array}$ & $\begin{array}{l}1.1 \\
1.8 \\
1.7 \\
2.0 \\
2.4 \\
2.7\end{array}$ & $\begin{array}{l}30.0 \\
\\
16.4 \\
21.2 \\
30.0\end{array}$ & $\begin{array}{l}2.8 \\
1.4 \\
1.3 \\
0.8 \\
0.7 \\
0.6\end{array}$ & $\begin{array}{r}23.0 \\
5.0 \\
5.0 \\
2.0 \\
2.5 \\
2.0\end{array}$ & $\begin{array}{r}17 \\
15 \\
12 \\
9 \\
6 \\
4\end{array}$ & $\begin{array}{l}1,376 \\
1,409 \\
1,677 \\
1,506 \\
1,367 \\
1,484\end{array}$ & $\begin{array}{r}98.3 \\
103.0 \\
101.0 \\
102.0 \\
100.0 \\
102.0\end{array}$ & $\begin{array}{r}83.0 \\
110.0 \\
96.0 \\
123.0 \\
139.0 \\
141.0\end{array}$ & $\begin{array}{l}111 \\
254 \\
230 \\
262 \\
261 \\
266\end{array}$ \\
\hline 13 & 21 & $\begin{array}{l}12 \\
18 \\
25 \\
33 \\
42 \\
60\end{array}$ & $\begin{array}{l}12 \\
18 \\
25 \\
33 \\
42 \\
60\end{array}$ & \begin{tabular}{|l|}
61.0 \\
61.2 \\
61.6 \\
62.4 \\
63.7 \\
65.1 \\
\end{tabular} & $\begin{array}{l}48 \\
48 \\
46 \\
44 \\
49 \\
49\end{array}$ & $\begin{array}{l}184 \\
175 \\
171 \\
171 \\
172 \\
173\end{array}$ & $\begin{array}{l}55 \\
46 \\
59 \\
54 \\
44 \\
40\end{array}$ & $\begin{array}{r}106 \\
88 \\
91 \\
97 \\
86 \\
79\end{array}$ & $\begin{array}{l}6.63 \\
6.61 \\
6.71 \\
7.39 \\
7.45\end{array}$ & $\begin{array}{l}3.83 \\
3.69 \\
4.39 \\
4.95 \\
4.87\end{array}$ & $\begin{array}{l}2.80 \\
2.92 \\
2.32 \\
2.44 \\
2.58\end{array}$ & $\begin{array}{l}1.4 \\
1.3 \\
1.5 \\
2.0 \\
1.9\end{array}$ & $\begin{array}{l}26.2 \\
37.5 \\
17.5\end{array}$ & $\begin{array}{l}8.9 \\
2.9 \\
1.6 \\
1.2 \\
0.7 \\
0.6\end{array}$ & $\begin{array}{r}37.5 \\
18.0 \\
2.0 \\
1.2 \\
2.0 \\
0.5\end{array}$ & $\begin{array}{l}8 \\
8 \\
8 \\
5 \\
6 \\
7\end{array}$ & $\begin{array}{r}725 \\
1,542 \\
1,710 \\
1,398 \\
1,384\end{array}$ & $\begin{array}{r}97.0 \\
98.0 \\
105.0 \\
104.0 \\
102.0 \\
102.0\end{array}$ & $\begin{array}{r}55.6 \\
78.8 \\
87.0 \\
88.0 \\
101.0 \\
100.0\end{array}$ & $\begin{array}{l}151 \\
248 \\
208 \\
210 \\
213 \\
206\end{array}$ \\
\hline 14 & 53 & $\begin{array}{l}19 \\
27 \\
35 \\
41 \\
47 \\
55 \\
62 \\
69 \\
76 \\
83\end{array}$ & $\begin{array}{l}14 \\
22 \\
30 \\
36 \\
42 \\
50 \\
57 \\
64 \\
71 \\
78\end{array}$ & \begin{tabular}{|l|}
80.1 \\
77.9 \\
75.6 \\
74.9 \\
75.6 \\
75.5 \\
76.1 \\
77.8 \\
77.8 \\
78.2
\end{tabular} & $\begin{array}{l}44 \\
43 \\
41 \\
41 \\
40 \\
41 \\
41 \\
41 \\
43 \\
44\end{array}$ & $\begin{array}{l}196 \\
198 \\
201 \\
188 \\
186 \\
189 \\
182 \\
183\end{array}$ & $\begin{array}{l}49 \\
43 \\
46 \\
53 \\
46 \\
45 \\
41 \\
39 \\
44 \\
36\end{array}$ & $\begin{array}{l}87 \\
76 \\
78 \\
91 \\
76 \\
77 \\
69 \\
66 \\
78 \\
65\end{array}$ & $\begin{array}{l}6.21 \\
6.11 \\
6.07 \\
6.03 \\
5.94 \\
6.77 \\
6.38 \\
5.52 \\
6.67 \\
6.54\end{array}$ & \begin{tabular}{|l|}
2.80 \\
2.79 \\
3.24 \\
3.53 \\
3.33 \\
4.01 \\
3.70 \\
3.71 \\
3.86 \\
3.95
\end{tabular} & $\begin{array}{l}3.41 \\
3.32 \\
2.83 \\
2.50 \\
2.61 \\
2.76 \\
2.68 \\
1.81 \\
2.81 \\
2.59\end{array}$ & $\begin{array}{l}0.8 \\
0.8 \\
1.1 \\
1.4 \\
1.3 \\
1.5 \\
1.4 \\
2.0 \\
1.4 \\
1.5\end{array}$ & $\begin{array}{l}31.2 \\
32.5 \\
\\
35.0 \\
21.2 \\
27.5 \\
28.7 \\
27.5 \\
31.2 \\
27.5\end{array}$ & \begin{tabular}{|r|}
23.2 \\
18.2 \\
13.0 \\
6.9 \\
4.6 \\
3.4 \\
2.5 \\
1.7 \\
1.4 \\
1.0
\end{tabular} & $\begin{array}{l}52.0 \\
49.0 \\
39.5 \\
27.5 \\
16.0 \\
17.0 \\
10.0 \\
10.5 \\
7.0 \\
6.5\end{array}$ & $\begin{array}{r}37 \\
30 \\
28 \\
27 \\
19 \\
17 \\
11 \\
11 \\
9 \\
9\end{array}$ & $\begin{array}{r}612 \\
522 \\
752 \\
703 \\
1,010 \\
1,048 \\
\\
1,062 \\
1,101\end{array}$ & $\begin{array}{r}98.2 \\
99.1 \\
101.0 \\
104.0 \\
103.0 \\
101.0 \\
104.0 \\
102.0 \\
103.0 \\
101.2\end{array}$ & $\begin{array}{r}21.0 \\
30.8 \\
82.0 \\
92.0 \\
103.0 \\
75.0 \\
50.0 \\
56.8 \\
67.0\end{array}$ & $\begin{array}{r}56 \\
78 \\
236 \\
225 \\
215 \\
237 \\
157 \\
168 \\
163\end{array}$ \\
\hline
\end{tabular}

* 5 mgm. per $\mathrm{kgm}$. of body weight; 45 -minute interval.

$\dagger$ Urine volume excreted during 4-hour period following ingestion of $1,500 \mathrm{ml}$. of water under basal conditions.

minute specimen), and the thymol turbidity reaction of the serum. These measurements and tests were performed each week and correlated with the above described body fluid measurements (Table I and Figure 3 ). The total plasma protein and albumin and globulin fractions were determined by a salting out procedure in which micro-digestion and Nesslerization were used (17). The plasma bilirubin method of Malloy and Evelyn (18) was adapted to the Coleman Jr. spectrophotometer, as was the bromsulfalein test (19) and the thymol turbidity reaction of the serum $(20,21)$. Blood chlorides were measured according to the methods of Wilson and Ball (22) on blood samples collected under oil. The same method was also applied to urine and all values were expressed as milliequivalents of chloride per liter.

In an attempt to reveal water storage and to give quantitative expression to the tendency for fluid retention, the patients under study were periodically "challenged" with a water tolerance test (23). Under basal conditions the patients drank $1,500 \mathrm{ml}$. of tap water in a 20 -minute period or less and the volume of urine excreted every half hour for the first hour and every hour thereafter for a total period of 4 hours was recorded. These tests were not undertaken on the days that the body fluid compartments were measured.

\section{RESULTS}

In Table I are shown the correlated data obtained in each case during simultaneous observations on body fluids, water tolerance, tests of liver function and measurements of the hematocrit, plasma proteins, and chlorides at periodic intervals in the course of acute hepatitis.

It is apparent that all patients exhibited greater thiocyanate spaces during the acute phase of hepatitis than later in the course of convalescence. The absolute and percentile increase in each case may 
be seen by reference to Table II. Although great individual variation is evident in the degree of change seen, the average expansion of this compartment over the lowest convalescent level was $24.5 \mathrm{ml}$. per $\mathrm{kgm}$. of body weight which represents a 13.5 per cent increase. With the exception of case 3, the plasma and blood volumes were also expanded during this period, less in absolute amount than the thiocyanate space, but greater when considered in terms of per cent of the lowest convalescent values. Although the average increase in plasma volume was $9.3 \mathrm{ml}$. per $\mathrm{kgm}$. of body weight, and the same value for blood volume was $18.9 \mathrm{ml}$. per $\mathrm{kgm}$., the per cent increase in these two compartments was 23.4 per cent and 27.1 per cent, respectively. Since these two figures indicate relatively identical percentage enlargements, it is evident that expansion of both the plasma and blood volumes during the acute phase of hepatitis occurs in a symmetrical manner. The increase in total circulating red cell volume, therefore, must keep pace with the swelling plasma volume, resulting in minimal alterations in the venous hematocrit throughout the course of illness. The acute phase of infectious hepatitis in comparison to convalescence is, therefore, char-

TABLE II

Tabular listing of 14 cases of acute infectious hepatitis to illustrate the absolute and percentile expansion of the thiocyanate space, plasma volume, and blood volume during the acute phase, with the lowest convalescent values as a base line

\begin{tabular}{|c|c|c|c|c|c|c|}
\hline \multirow{2}{*}{$\begin{array}{c}\text { Case } \\
\text { number }\end{array}$} & \multicolumn{2}{|c|}{$\begin{array}{l}\text { Increase in } \\
\text { thiocyanate space }\end{array}$} & \multicolumn{2}{|c|}{$\begin{array}{c}\text { Increase in } \\
\text { plasma volume }\end{array}$} & \multicolumn{2}{|c|}{$\begin{array}{l}\text { Increase in } \\
\text { blood volume }\end{array}$} \\
\hline & & Increase & & Increase & & Increase \\
\hline $\begin{array}{r}\mathbf{1} \\
2 \\
3 \\
4 \\
5 \\
6 \\
7 \\
8 \\
9 \\
.10 \\
11 \\
12 \\
13 \\
14\end{array}$ & $\begin{array}{c}\text { ml. per } \\
\text { kgm. } \\
30 \\
21 \\
21 \\
28 \\
56 \\
12 \\
17 \\
40 \\
27 \\
25 \\
37 \\
2 \\
13 \\
14\end{array}$ & $\begin{array}{c}\text { per cent } \\
17.7 \\
10.7 \\
14.2 \\
16.5 \\
27.7 \\
7.1 \\
10.7 \\
19.5 \\
14.1 \\
11.4 \\
22.7 \\
1.0 \\
7.6 \\
7.7\end{array}$ & \begin{tabular}{|c} 
ml. per \\
kgm. \\
10 \\
18 \\
$-5^{*}$ \\
12 \\
7 \\
20 \\
8 \\
8 \\
5 \\
9 \\
5 \\
5 \\
15 \\
13
\end{tabular} & \begin{tabular}{|c} 
per cent \\
33.0 \\
48.0 \\
$-10.0 *$ \\
29.3 \\
19.2 \\
52.6 \\
9.5 \\
22.9 \\
12.8 \\
22.5 \\
13.9 \\
12.7 \\
37.2 \\
36.1
\end{tabular} & \begin{tabular}{|c|} 
ml. per \\
kgm. \\
23 \\
40 \\
$-13^{*}$ \\
25 \\
18 \\
40 \\
17 \\
17 \\
7 \\
18 \\
14 \\
9 \\
27 \\
22
\end{tabular} & \begin{tabular}{|c} 
per cent \\
37.1 \\
59.8 \\
$-12.5^{*}$ \\
30.6 \\
26.6 \\
54.8 \\
22.7 \\
25.7 \\
9.2 \\
25.0 \\
20.2 \\
12.0 \\
34.2 \\
34.1
\end{tabular} \\
\hline $\begin{array}{l}\text { verage } \\
\text { crease }\end{array}$ & 24.5 & 13.5 & 9.3 & 23.4 & 18.9 & 27.1 \\
\hline
\end{tabular}

* Decrease.

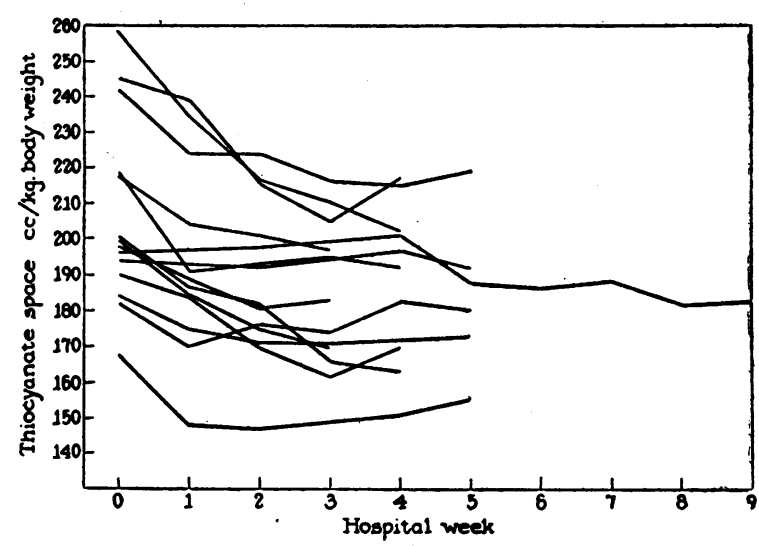

Fig. 1. Alterations in Thiocyanate Space in 14 Patients During the Course of Acute Infectious Hepatitis

acterized by an increase in both total red cell volume and plasma volume [polycythemic hypervolemia (38)].

The rate of fall in the individual fluid compartments with recovery may be seen by reference to the individual columns in Table I. The composite values for the thiocyanate space throughout the course of illness are graphically represented in Figure 1. The rate of fall in this compartment was greatest during the first week of hospitalization and was usually detectable by a loss in body weight quite comparable to the amount of fluid lost by reduction in the size of this compartment. When mobilization of this fluid space occurred rapidly, a detectable diuresis appeared (Figure 3 ). Despite the demonstration of an expanded thiocyanate compartment in every case studied, edema and ascites were never clinically apparent.

The variable urine volume following ingested water during these periods was of particular interest. The water tolerance test previously described yielded progressively larger excretory volumes during the course of convalescence. The greatest increase in the output of urine following the $1,500 \mathrm{ml}$. drink of water occurred when the thiocyanate space was maximally contracting and was thought to indicate a decreasing tendency to retain water. The excretion patterns obtained at different periods of illness were quite distinct. An example of delayed urine output following the test dose of water given during the most acute phase of illness is illustrated in the slowly rising pattern of Figure 2A (case 10). In this instance 

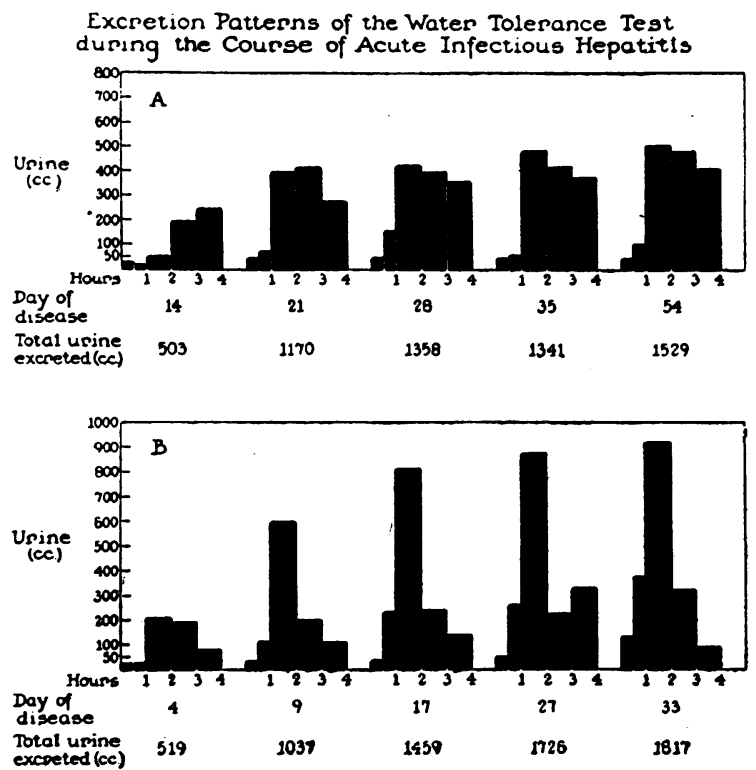

Fig. 2. Excretion Patterns of the Water Tolerance Test During the Course of Acute Infectious HePatitis

there was a step-wise increase in urine volume over the 4-hour period of observation. Later in convalescence larger amounts of urine were excreted in the earlier time periods of the test and a "shift to the left" occurred in the pattern (23). The total urine volume of the test period likewise increased with convalescence. The peak of excretion during the recovery period was usually reached in the second hour of the test. In many cases these peaks were conspicuous (Figure 2B, Case 3 ) and increased in size as the patient recovered. With the adjustment of the thiocyanate space to base line convalescent levels, little tendency to retain ingested water was noted as measured by the water tolerance test and almost the entire volume of ingested water was excreted within the 4-hour period and often surpassed.

Tests of liver function applied during the observations of fluid shift indicated progressive restoration of function. The extent of hepatic control over the dynamics of this shifting fluid pattern may be appreciated from the fact that the largest movements in body fluid occurred during those periods of greatest improvement in hepatic function and were most apparent during the first two weeks of hospitalization. Following this period, both hepatic function and fluid shift moved less rapidly towards stabilization.
A slight depression of plasma protein concentration was observed during the acute phase of illness that was undoubtedly due in part to the hypervolemia and in part to nutritional factors. These changes were of too little magnitude to have been influential in affecting the fluid movements described above. However, a secondary rise in plasma volume was noted in cases $2,3,7,12,13$ and 14 during the second week of recovery which often approached the level of the plasma volume obtained during the early acute phase of the disease. This may have been due to the slight but inconstant rise in the total circulating albumin which occurred at this time, possibly on the basis of enhanced nutrition and improvement in albumin synthesis by the liver.

The behavior of the plasma and urine chlorides during the course of acute hepatitis was thought to be of importance because of the previous demonstration of chloride retention in cirrhosis and liver atrophy $(24,25)$, sodium retention in "catarrhal" jaundice (26), and disturbance in the metabolism of inorganic salts of the blood and tissue in various hepatic diseases (27). In addition, it had been shown that depressed plasma and urine chloride levels develop in animals following acute liver injury by arsphenamine (28).

On admission, the majority of patients revealed low plasma chlorides varying from 90 to 98 meq. per liter. The urine chloride concentration and total 24-hour urine chloride excretion were similarly depressed at this early period. With recovery and mobilization of the expanded thiocyanate space, the plasma chlorides rose and increasing amounts of chloride appeared in the urine. This rise could not be explained wholly by the chloride intake of the patient which was kept constantly between 9 and 10 grams daily, because it often occurred several days after the beginning of the constant salt intake. More often these adjustments awaited the contraction of the thiocyanate fluid compartment. In the presence of an increased thiocyanate compartment, as well as an increased plasma and blood volume, it was difficult to attribute the low urine volume, depressed urine chlorides, and tendency for water storage to simple dehydration (29). It was believed on the basis of these observations, therefore, that increased amounts of chloride were held in the expanded thiocyanate compartment during the acute stage of 
hepatitis, in compliance with the laws of diffusion equilibria, and that with contraction of this compartment during recovery, increased quantities of chloride were mobilized and appeared in greater amounts in both plasma and urine.

Presentation of data from case 10 (Figure 3) with a description of the course of the disease will serve to correlate more clearly the movements of body fluids, the tendency to store fluid, the alterations in blood constituents, and the state of liver function during the course of acute infectious hepatitis.

F. J. E., a 34-year-old naval lieutenant commander, was admitted to the Hospital of The Rockefeller Institute on March 24, 1946, complaining of jaundice. Twelve days before admission he had developed a mild headache followed by anorexia, malaise, generalized muscular aches, and lassitude. He went to bed and felt slightly better after 3 days of rest, fluids, and a moderate food intake. During this time light tan stools were passed and 2 days later darkening of the urine appeared. By March 22, ic-

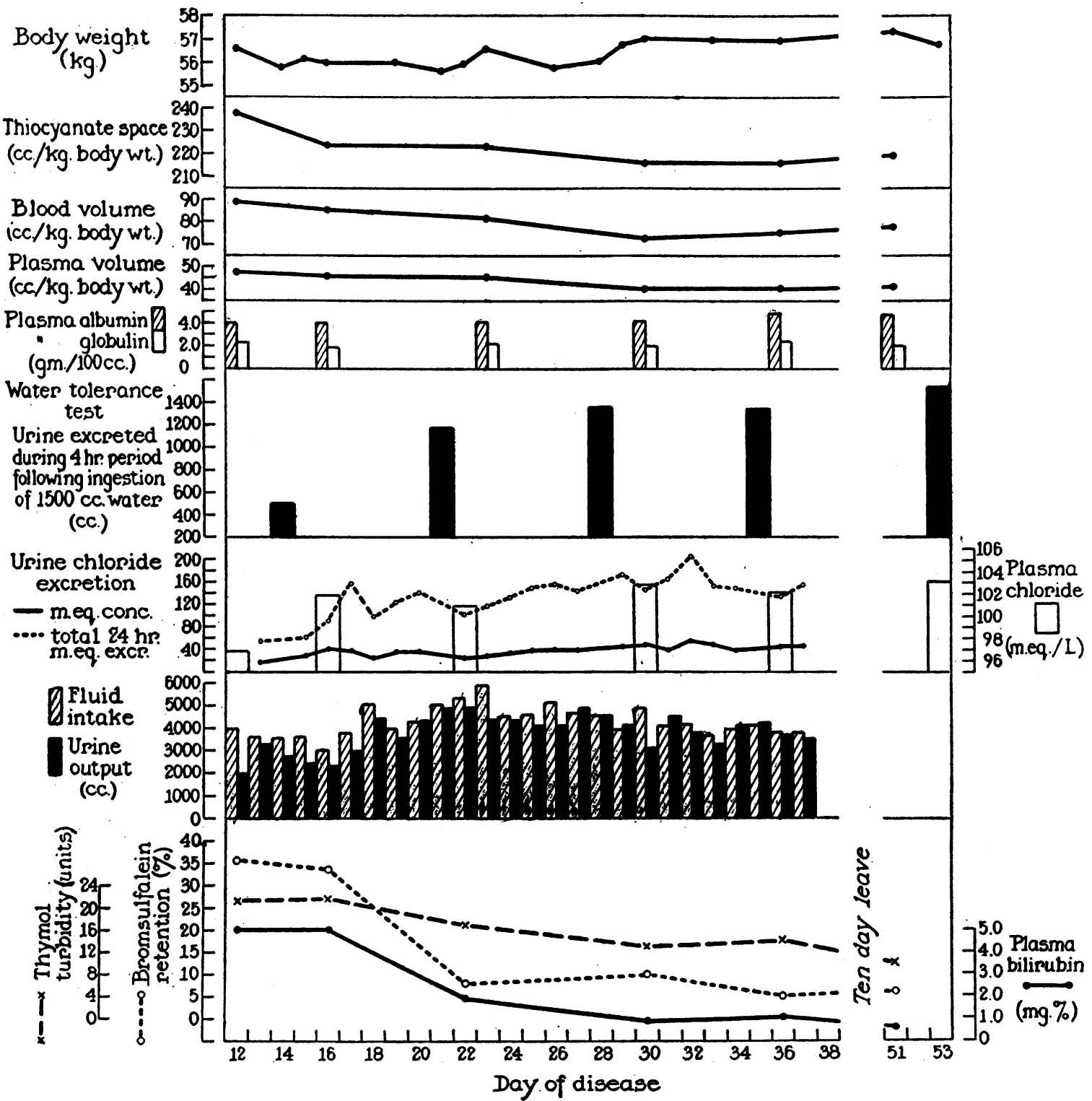

Fig. 3. The Data from Case 10 Correlating the Alterations of the Body Fluid Compartments, Water Tolerance, Blood Constituents, Chlorides and Water Balance with Recovery of Liver Function During the Course of Acute Infectious Hepatitis 
terus of the sclerae was noted but he was better able to eat and maintain his fluid intake. There was no history of previous illness, transfusions, inoculations or exposure to toxic chemical agents. One month before onset, however, the patient's fiancée had developed acute infectious hepatitis and was moderately ill for a period of 10 days. Physical examination of the patient on admission revealed a lean, muscular, adult male with a rectal temperature of $99.2^{\circ} \mathrm{F}$; he appeared to be moderately ill. There was marked icterus of the skin and sclerae. No rash or spider angiomata was discernible. The edge of the liver was felt $1.0 \mathrm{~cm}$. below the costal margin and was tender; the tip of the spleen descended one finger's breadth below the left costal margin. There was no apparent edema or ascites. The remainder of the physical examination was negative.

Laboratory data on admission revealed a hematocrit of 46, normal blood counts, a negative urine examination, urine specific gravity 1.010 , serum bilirubin of $5 \mathrm{mgm}$. per cent, bromsulfalein retention of 35.5 per cent, and a thymol turbidity reaction of 21.3 units. The total plasma proteins were 6.25 grams per cent, albumin 3.97 grams per cent and globulin 2.28 grams per cent with an $A / G$ ratio of 1.7 .

The patient was given the diet and salt intake previously prescribed for this group and careful measurements of fluid intake and urine output were begun.

The patient was completely afebrile while in the hospital. Following the institution of bed rest and adequate nutrition, recovery proceeded without complication. The urine became lighter, increased color appeared in the stool, icterus diminished and disappeared. The liver and spleen receded in size, finally becoming impalpable.

The initial measurements of the body fluid compartments were undertaken the day following admission. The first 2 hospital days were marked by a fall in body weight amounting to 0.8 kilogram. Parallel with this, the thiocyanate space contracted from $237 \mathrm{ml}$. per $\mathrm{kgm}$. of body weight to $223 \mathrm{ml}$. per $\mathrm{kgm}$. of body weight. This represented a contraction of $14 \mathrm{ml}$. per $\mathrm{kgm}$. of body weight or a total of $784 \mathrm{ml}$. which could quite accurately account for the drop in body weight described above if this fluid volume was simultaneously excreted. A slight decline in blood and plasma volume accompanied these changes. During convalescence there was marked improvement in the tests of liver function and the final value for the thiocyanate space was $215 \mathrm{ml}$. per $\mathrm{kgm}$. after recovery was complete.

In spite of a fluid intake of $3,000 \mathrm{ml}$. to $4,000 \mathrm{ml}$. from the time of admission, the urine volume remained small. In addition, the plasma and urine chloride concentrations remained low. The water tolerance test, performed on the third hospital day, yielded only $503 \mathrm{ml}$. of urine following the ingestion of $1,500 \mathrm{ml}$. of tap water. By the 17 th day of illness, when marked improvement in liver function was evident, there appeared an increase in urine output which approximated 85 per cent to 90 per cent of the fluid intake for any given day. Although the salt intake had been maintained between 9 and 10 grams since admission, chlorides did not appear in the urine in increased amounts until this period of diuresis. On the 21st day of illness, $1,170 \mathrm{ml}$., or more than double the previous urine volume, was excreted during the water tolerance test. Subsequent tests showed slight increase over this value (Figure 2A). For the remainder of the period of study the daily urine volume remained at normal levels. A weight gain appeared in the mid-period of recovery which was probably a result of improvement in nutrition and liver function. By the time of discharge from the hospital the gain in body weight had been held, liver function had been adequately restored, and recovery was considered complete. In addition, a 10-day leave period had been tolerated without difficulty. The minimal fluctuations in plasma protein concentration noted during the course of illness bore no constant relation to the alterations of body fluids described above. Throughout the period of hospitalization, dehydration, edema, and ascites had never become clinically evident.

\section{DISCUSSION}

The expanded thiocyanate compartments and the relatively poor water tolerance of patients during the acute phase of infectious hepatitis substantiate the concept of Jones and Eaton (6) that increased water storage occurs during this period of the disease. It was also their suggestion that the diuresis of convalescence resulted from improved hepatic efficiency, and that this resulted in a "shift of fluid from the tissues or serous cavities to the blood stream with the ultimate establishment of diuresis." In keeping with these suggestions, the shifts of body fluid, as demonstrated by the present study with methods that offer quantitation of individual fluid compartments, reveal shrinkage of the thiocyanate compartment during recovery with an accompanying rise in urine output. The tendency to retain water during acute hepatic in- 
sufficiency, as expressed by the water tolerance test, has been noted to occur in infectious hepatitis (23) as well as in acute liver injury produced by chemical substances $(28,30)$. In the partially hepatectomized dog, hydrothorax may develop within a few days of operation, often of sufficient amount to drown the animal (31). Ascites may also appear despite the presence of an Eck fistula. In addition, total extirpation of the liver in frogs has been shown to result in tremendous edema (32) and decreased water tolerance (33). The oliguria and anuria of yellow fever are reported to be most severe in those cases in which there is a correspondingly great amount of hepatic necrosis (34). It has been suggested that the increased retention of water resulting in hyperhydration of the body because of damage to organs such as the liver might serve a useful purpose in diluting and overcoming the harmful effects of too great a concentration of injurious substances formed during infection (35). Similar water retention phenomena have been produced by dietary liver injury as demonstrated by the experiment in which rats kept on a high fat diet when dehydrated retained more from a given quantity of water than did normally fed animals under the same conditions (36).

The expansion of the thiocyanate space during the acute phase of hepatitis, together with the enlarged plasma volume, signifies a general distension of all the extravascular spaces. The minimal change in the venous hematocrit noted throughout the course of hepatitis, despite the increased plasma volume, must indicate a symmetrical enlargement of the total red cell volume. However, the slight reticulocytosis during this period of the disease could not account for the increase in the red blood cell volume on the basis of the formation of new red cells, although extramedullary hematopoiesis has been reported in fatal cases of acute liver disease (37). A partial explanation may reside in the fact that the acutely inflamed liver fails to store blood efficiently with the result that more blood remains in the general circulation $(38,39)$. During the acute stage of hepatitis, wide-spread edema and necrosis occupy much of the liver substance $(40,41)$ which, under these circumstances, probably contains relatively less blood. In addition, sinusoidal congestion is more evident during the healing phase of hepatis. Although there is no information available relating to the hepatic blood flow and total blood contained within the liver during acute infectious hepatitis, it is not unreasonable to consider that acute inflammatory disease of the liver might shunt large amounts of blood to the general circulation. Simple hypervolemia has been reported in cirrhosis of the liver $(42,43)$.

A partial explanation for the maintenance of the hematocrit in the face of an expanded plasma volume might be found in the slight macrocytosis of acute hepatitis. Variations in the Mean Corpuscular Volume of from $95 \mathrm{cu} . \mu$ to $120 \mathrm{cu} . \mu$ and in the Mean Corpuscular Hemoglobin Concentration of from 28 per cent to 35 per cent, have been encountered in hepatitis $(44,45)$, suggesting that the red cells may be swollen during the acute phase of jaundice but gradually return to normal size with recovery of the patient.

The results of the investigations cited above have served to indicate in outline the conditions under which water retention may occur in the presence of diminished liver function, and have pointed out the possible factors believed to be responsible for the expansion of the plasma and blood volumes during acute hepatitis. There remain to be defined the forces underlying this shifting fluid pattern.

The exact role of the kidney in relation to these movements of fluid in liver disease has never been established. While the "hepato-renal" syndrome clinically suggests that disastrous disturbances in water balance may occur under combined hepatic and renal control, biochemical and metabolic studies of this state have failed to disclose what fundamental mechanisms are aberrant $(46,47)$.

The fluid content of the tissues has been considered to be under hepatic control by virtue of metabolic (48) and hormonal regulation (49, 50, 51). Relevant to this concept are the unconfirmed data indicating that the acute phase of hepatitis is accompanied by an increase in the urinary excretion of biologically active estrogen (52). The water-storing influence of active estrogen is well recognized and is commonly encountered in the premenstrual state when there may occur edema, swelling of the breasts, and a gain in body weight (53).

A recent report (54) indicated that the urine of patients with cirrhosis and ascites, when injected into hydrated rats, delayed the excretion of urine. 
When the urine of cirrhotic patients in whom ascites had been controlled was injected, it was found to have antidiuretic activity greater than normal urine but less than urine from ascitic patients. In 6 patients with cirrhosis, re-accumulation of ascitic fluid ceased with dietary therapy and intravenous liver extract before demonstrable increase in the plasma albumin levels. This experience has been confirmed (55) and emphasizes the importance of factors other than the serum proteins that may have influence over water storage during liver disease. This has been a matter of comment in reference to the general problem of edema for many years (56).

The serum protein concentration and total circulating protein exhibited little alteration during the course of acute hepatitis in the 14 patients in the present study and bore no constant relation to the changes in plasma volume. The quantitative changes in the plasma proteins that may occur independent of alterations in the plasma volume have been reported experimentally and are to be found especially where nutritional features are of great importance (57). The results of the present work, therefore, suggest that factors other than the simple effect of the serum proteins account for water storage and decreased water tolerance during the acute phase of jaundice and the movement of water from the interstitial fluid spaces with recovery. Reference to the antidiuretic principle, therefore, seems to be pertinent. The pituitary origin of this substance has been suggested (58, 59) but requires further elucidation. Heller and Urban (60) have demonstrated that normal liver in vitro possesses great capacity to adsorb (inactivate) the antidiuretic principle of the posterior lobe of the pituitary when compared with blood, muscle, brain and kidney. Boiled liver tissue, however, was completely ineffective. In addition, following the intravenous injection of large doses of pituitrin, there was rapid disappearance of antidiuretic activity from the circulating blood. These experimental data suggest that the normal liver inactivates the antidiuretic principle and thereby maintains normal diuresis and body water content. In acute hepatitis it is conceivable, therefore, that with diffuse hepatic damage and depression of liver function, this inactivation is incompletely performed and water retention results. With recovery and improvement in hepatic efficiency, in- activation of the antidiuretic principle is resumed, resulting in diuresis and shrinkage of the thiocyanate space to a normal volume. Bioassays of the antidiuretic substance in the urines of patients with acute infectious hepatitis are now under way in this laboratory.

\section{SUM MARY}

1. Periodic observations of the thiocyanate space, total blood and plasma volumes were undertaken during the hospital course of 14 patients with acute infectious hepatitis under conditions of rigid water balance and correlated with measurements of plasma and urine chlorides, the tendency to retain ingested water as measured by a water tolerance test, and tests of liver function.

2. During the acute phase of hepatitis, the thiocyanate compartment and blood and plasma volumes were found to be expanded. Depressed plasma and urinary chlorides as well as an increased tendency to retain ingested water accompanied these changes.

3. With convalescence and improving liver function, shrinkage of the thiocyanate compartment and a decrease in the total blood and plasma volumes occurred. A diuresis and decreased tendency to retain ingested water were noted at this time and a rise in plasma and urinary chloride levels followed.

4. The slight alterations in total circulating protein were thought to be attributable to nutritional factors and to decreased protein synthesis during hepatitis and were not considered to be of major importance in determining these fluid shifts.

5. It is suggested that these movements in body fluids during acute infectious hepatitis may be directed by endocrine factors and related to incomplete inactivation of the antidiuretic principle by a damaged liver. ${ }^{2}$

\footnotetext{
2 Recent studies by Shorr, Zweifach and Furchgott (Science, 1945, 102, 489) have indicated that a vasodepressor substance of hepatic origin (VDM) is associated with the opening up of the capillary bed during the decompensatory stage of experimental shock. More recently, in studies with Furth and Sobol, they have also been able to detect VDM in the blood of mice with granulosa cell tumors, concurrent with the development of hypervolemia in these animals (Science, 1947, 105, 41). VDM was also shown by them to appear in the blood of human beings with liver cirrhosis during periods of hepatic decompensation and in the blood and liver wash of rats with experimental hepatic cirrhosis. Finally; a
} 
The advice and guidance of Dr. D. D. Van Slyke is gratefully acknowledged.

\section{BIBLIOGRAPHY}

1. Gilbert, A., and Lereboullet, P., Des urines retardées (opsiurie) dans les cirrhoses. Compt. rend. Soc. Biol., 1901, 53, 276.

2. Van Noorden, C., Metabolism and practical medicine. Pathology, 1907, 2, 267.

3. Steinthal, Ueber Icterus Gravis und Anure. Beitr. klin. Chir., 1911, 76, 629.

4. Whitla, W., Uremia in affections of the liver. Dublin J. Med. Sci., 1876, 61, 107.

5. Adler, A., Liver and diuresis. Klin. Woch., 1923, 2, 1980.

6. Jones, C. M., and Eaton, F. B., The prognostic significance of a spontaneous diuresis in acute or subacute disease of the liver. New England J. Med., 1935, 213, 907.

7. Rutstein, D. D., Thomson, K. J., Tolmach, D. M., Walker, W. H., and Floody, R. J., Plasma volume and "extravascular thiocyanate space" in pneumococcus pneumonia. J. Clin. Invest., 1945, 24, 11.

8. Labby, D. H., and Hoagland, C. L., Alterations in the body fluids during acute infectious hepatitis. Proc. Soc. Exper. Biol. and Med., 1946, 63, 110.

9. Hoagland, C. L., and Shank, R. E., Infectious hepatitis; Review of 200 cases. J. A. M. A., 1946, 130, 615.

10. Kunkel, H. G., Labby, D. H., and Hoagland, C. L., Chronic liver disease following infectious hepatitis. I. Abnormal convalescence from initial attack. Ann. Int. Med. (In press.)

11. Gregersen, M. I., and Stewart, J. D., Simultaneous determination of the plasma volume with $\mathrm{T}-1824$ and the "available fluid" volume with sodium thiocyanate. Am. J. Physiol., 1939, 125, 142.

12. Phillips, R. A., A method for the determination of the blue dye T-1824 in plasma. J. Exper. Med., 1943, 77, 421.

13. Gibson, J. G., Jr., and Evans, W. A., Jr., Clinical studies of blood volume; clinical application of method employing azo dye "Evans blue" and spectrophotometer. J. Clin. Invest., 1937, 16, 301.

14. Wintrobe, M. W., Clinical Hematology. Lea and Febiger, Philadelphia, 1942.

15. Keith, N. M., Rowntree, L. G., and Geraghty, J. T., A method for the determination of plasma and blood volume. Arch. Int. Med., 1915, 16, 547.

highly purified VDM concentrate, prepared from beef liver by Mazur in their laboratory, was found to exert a profound anti-diuretic effect in the normal dog on intravenous administration. These observations have led these investigators to suggest the causal relation between the hepatic vaso-depressor, VDM, and the increased blood volume and the anti-diuresis of hepatic cirrhosis and infectious hepatitis. (Personal communication.)
16. Gibson, J. G., 2nd, and Evans, W. A., Jr., Clinical studies of the blood volume. II. The relation of plasma and total blood volume to venous pressure. blood velocity, rate, physical measurements, age and sex in ninety normal humans. J. Clin. Invest. 1937, 16, 317.

17. Archibald, R. M., Army Medical Laboratory Manual. 1947. To be published.

18. Malloy, H. T., and Evelyn, K. A., The determination of bilirubin with the photoelectric colorimeter. J. Biol. Chem., 1937, 119, 481.

19. Mateer, J. G., Baltz, J. I., Marion, D. F., and MacMillan, J. M., Liver function tests., J. A. M. A., 1943, 121, 723.

20. Shank, R. E., and Hoagland, C. L., A modified method for the quantitative determination of the thymol turbidity reaction of serum. J. Biol. Chem., 1946, $162,133$.

21. Kunkel, H. G., and Hoagland, C. L., Persistence of elevated values for the thymol turbidity test following infectious hepatitis. Proc. Soc. Exper. Biol. and Med., 1946, 62, 258.

22. Wilson, D. W., and Ball, E. G., A study of the estimation of chloride in blood and serum. J. Biol. Chem., 1928, 79, 221.

23. Aldersberg, D., and Fox, C. L., Changes of the water tolerance test in hepatic disease. Ann. Int. Med., 1943, 19, 642.

24. Kriss, B., and Pollak, L., Ueber die Chlorausscheidung bei Leberkranken nach Belastung mit Verschiedenen Chloriden. Wien. klin. Wchnschr., 1927, 40, 1251.

25. Thiers, H., A propos des syndromes chloropéniques. Les chloropénies sans azotémie et les chloropénies cirrhotiques. Etat du chlore tissulaire. J. de Physiol. et de Path. Gén., 1930, 28, 86.

26. Siedek, H., and Zuckerkandl, F., Der Natrium-Chlor Quotient bei Icterus Catarrhalis. Klin. Wchnschr., 1935, 14, 568.

27. Shigemi, H., . Studies on metabolism of inorganic salts and water in hepatic disturbances. Jap. J. Gastroenterol., 1935, 7, 12.

28. Soffer, L. J., Dantes, A. D., and Sobotka, H., Electrolytes of blood and urine of dogs with acute hepatic injury produced by arsphenamine. Arch. Int. Med., 1937, 60, 509.

29. Lyons, R. H., Jacobson, S. D., and Avery, N. L., Jr., The effect on the plasma volume of dehydration produced by a low salt diet and ammonium chloride. Am. Heart J., 1944, 27, 353.

30. Aldersberg, D., and Paul, B., Ueber Veraenderungen des Wasserhaushaltes im Anaphylaktischen und im Histaminschock. Ztschr. f. exper. Med., 1933, 92, 293.

31. Mann, F. C., The relation of the liver to metabolism. Harvey Lectures, New York, 1927, $23,49$.

32. Frohlich, A., and Zak, E., Der Kreislauf in der Froschzunge unter pathologischen Bedingungen. Klin. Wchnschr., 1922, 1, 1055. 
33. Molitor, H., and Pick, E. P., Die Bedeutung der Leber für die Diurese. Arch. f. exp. Pathologie und Pharmakologie, 1923, 97, 317.

34. Soper, F. L., The pharmacopeia and the physician, treatment of yellow fever. J. A. M. A., 1942, 118, 374.

35. Krieger, V. I., and Kilvington, T. B., Antidiuretic substance in urine in relation to normal and toxaemic pregnancy. Med. J. Australia, 1940, 1, 575.

36. Shay, H., Kohm, R., and Fels, S. S., Some observations on changes in renal water output of the albino rat under the influence of a high fat diet. Gastroenterology, 1945, 4, 257.

37. Wintrobe, M. W., Relation of disease of the liver to anemia; type of anemia, response to treatment and relation of type of anemia to histopathologic changes in liver, spleen and bone marrow. Arch. Int. Med., 1936, 57, 289.

38. Best, C. H., and Taylor, N. B., The Physiological Basis of Medical Practice. 4th Ed., The William Wilkins \& Co., Baltimore, Md., 1945.

39. Barcroft, J., Nisimaru, Y., and Ray, G. B., Observations on the time taken for corpuscles to traverse the liver. J. Physiol.; 1932, 74, 44.

40. Roholm, K., and Iversen, P., Histopathology of acute infective hepatitis, changes in the liver in acute epidemic hepatitis (catarrhal jaundice) based on 38 aspiration biopsies. Acta Path. et Microbiol. Scandinav., 1939, 16, 427.

41. Lucké, B., and Mallory, T., The fulminant form of epidemic hepatitis. Am. J. Path., 1946, 22, 867.

42. Perera, G. A., The plasma volume in Läennec's cirrhosis of the liver. Ann. Int. Med., 1946, 24, 643.

43. Labby, D. H., The relation of plasma volume to the serum proteins in diseases of the liver. In preparation.

44. Hill, J. N., and Hausman, W., Liver deficiency anae$\mathrm{mia}$ in a case of acute infective hepatitis. Brit. $\mathrm{M}$. J., 1943, 2, 262.

45. Labby, D. H., Kunkel, H. G., Shank, R. E., and Hoagland, C. L., Metabolic and therapeutic experiences in acute hepatic insufficiency: A study of 386 cases of acute infectious hepatitis. In preparation.

46. Lichtman, S. S., and Sohval, A. R., Clinical disorders with associated hepatic and renal manifestations, with especial reference to the so-called "hepato-renal syndrome." Am. J. Digest. Dis. and Nutrition, 1937, 4, 26.

47. Boyce, F. F., The Role of the Liver in Surgery. Charles C. Thomas, Baltimore, Maryland, 1941.

48. Gerritzen, F., Liver diuresis. Acta med. Scandinav., 1936, 89, 101.

49. Pick, E. P., The regulation of water metabolism. Harvey Lectures, New York, 1929, 25, 25.

50. Aldersberg, D., Zur Rolle der Leber im Wasserhaushalt. Wien. Arch. f. inn. Med., 1943, 25, 269.

51. Pick, E. P., Topics in the study of water metabolism. J. of Mt. Sinai Hosp., 1946, 13, 167.

52. Gilder, H., and Hoagland, C. L., Urinary excretion of estrogens and 17-ketosteroids in young, adult males with infectious hepatitis. Proc. Soc. Exper. Biol. and Med., 1946, 61, 62.

53. Robinson, F. H., and Farr, L. E., The relation between clinical edema and the excretion of an antidiuretic substance in the urine. Ann. Int. Med., 1940, 14, 42.

54. Ralli, E. P., Robson, J. S., Clarke, D. H., and Hoagland, C. L., Factors influencing ascites in patients with cirrhosis of the liver. J. Clin. Invest., 1945, 24, 316.

55. Labby, D. H., Shank, R. E., Kunkel, H. G., and Hoagland, C. L., The treatment of cirrhosis of the liver with intravenous crude liver extract. J. A. M. A. (In press.)

56. Fremont-Smith, F., The mechanism of edema formation. New England J. Med., 1932, 206, 1286.

57. Mellors, R. C., Muntwyler, E., Mautz, F. R., and Abbott, W. E., Changes of the plasma volume and "available (thiocyanate) fluid" in experimental dehydration. J. Biol. Chem., 1942, 144, 785.

58. Gilman, A., and Goodman, L., The secretory response of the posterior pituitary to the need for water conservation. J. Physiol., 1937, 90, 113.

59. Ingram, W. R., Ladd, L., and Benbow, J. T., The excretion of an antidiuretic substance and its relation to the hypo-thalamicohypophyseal system in cats. Am. J. Physiol., 1939, 127, 544.

60. Heller, H., and Urban, F. F., The fare of antidiuretic principle of post pituitary extracts in vivo and in vitro. J. Physiol., 1935, 85, 502. 